\title{
HF Frequency Short-Term Forecast Method Research in the Asia Oceania Region
}

\author{
Chao $\mathrm{Li}^{1}$, Hanguang $\mathrm{Jia}^{1}$, Huibin $\mathrm{Hu}^{2}$, Yunjiang Liu ${ }^{1}$ and Xiangyang $\mathrm{Ye}^{1}$ \\ ${ }^{1}$ Institute of information and Navigation, Airforce Engineering University, Xi'an, China \\ ${ }^{2}$ The Chinese People's Liberation Army 94860 force, Nanjing, China
}

\begin{abstract}
With analysis on ionosphere propagation features, and on the basis of ionospheric data in Sanya, Hainan, the selfcorrelation coefficient analysis method is applied to the ionospheric parameter prediction, then the method is compared with the artificial neural network prediction method. The prediction of the autocorrelation coefficients method is more close to the actual value, and more accurate scientific basis is provided for the HF frequency prediction.
\end{abstract}

Keywords-autocorrelation coefficient; artificial neural network; foF 2

\section{INTRODUCTION}

HF communication is one of the main means of remote communication, and play a strong role in communication distance and maneuverability. Because propagation of sky waves mainly depends on ionosphere reflection, and propagation parameters have the characteristics of time-varying dispersive, so the frequency is unpredictable and communication performance declines. Ionospheric variability is very acute and abnormal in the Asia Oceania region, especially for the South China Sea with "ionospheric gradients" and "double hump" phenomenon, so how to predict HF frequency accurately is always one of the research hotspot.

The prediction of ionospheric parameters is the critical point of HF frequency prediction. ITU-RP.1239 method is adopted in the REC533 model recommended by ITU to predict ionospheric parameters, and the digital map data in the Chinese region is deduced from the ionospheric data of few observation station, the predicted value compared with the actual value is still a gap. The Asia-Oceania method is a calculation model to predict ionospheric parameters in Asia Oceania region, and the method proves that the correlation in between ionospheric parameters and forecast index(Ic) are better than in between ionospheric parameters and sunspot number(R12); but ionospheric acute movements in the South China Sea has been concentrated less, and the method is mainly used to mid-andlong term forecasting. At present, short-term forecast methods of ionospheric parameters at home and abroad include autocorrelation analysis ${ }^{[1]}$, multiple source linear regression ${ }^{[2-4]}$, artificial neural network ${ }^{[5-7]}$ and similar day method ${ }^{[8-9]}$, and so on. Various predicted methods produce different predicted results, and each has merits and defects.

With analysis on ionosphere propagation features in Sanya, Hainan, the autocorrelation coefficient analysis method is applied to the critical frequency's (foF2) Short-Term prediction, then the predicted value is compared with the artificial neural network prediction method's. The conclusion could be drowned, that more accurate predicted value will be acquired by the autocorrelation coefficients method.

\section{AutOcoRRELATION COEFFICIENTS UnSTABLE PREDICTED METHOD}

For a random process $\mathrm{x}(\mathrm{t})$, which statistical characteristics can usually be described by mathematic expectation, variance and autocorrelation coefficients in probability theory. When second order stationarity is meet, the $\mathrm{x}(\mathrm{t})^{\prime}$ 's regularities of distribution will not change over time. Its definity is

$$
\begin{gathered}
\mathrm{m}=\mathrm{E}[x(\mathrm{t})] \\
\sigma=\sqrt{E\left[(\mathrm{x}(\mathrm{t})-\mathrm{m})^{2}\right]} \\
\mathrm{p}(\tau)=\frac{E\left[x\left(\mathrm{t}_{2}\right) x\left(\mathrm{t}_{2}\right)\right]-\mathrm{m}^{2}}{\sigma^{2}}
\end{gathered}
$$

The mathematic expectation $\mathrm{m}$ is described as the average of $x(t)$, and the variance $\sigma$ is expressed as the change intension of $x(t)$ around the average value, and the $\rho(t)$ is defined as the $x(t)$ 's autocorrelation coefficient with the value range from -1 to +1 . The $\rho(t)$ is described as linear dependence relation between two random variables $x(t)$ and $x(t+\tau)$ in time $t$ and $t+\tau$, and the greater the correlation index the greater will be the linear relation.

In practice, the number of the sample is limited, then suppose that $x\left(t \_p\right)$ is measured value of $x(t)$ in time $t \_p$ $(\mathrm{p}=0,1,2, \ldots, \mathrm{N}-1)$. So practical design formulas could be acquired:

$$
E[x(t)]=\frac{1}{N} \sum_{p=0}^{N-1} x\left(t_{p}\right)
$$

$$
\sigma=\sqrt{\frac{1}{N} \sum_{p=0}^{N-1}\left(x\left(t_{p}\right)-\overline{x(t)}\right)^{2}}
$$




$$
\begin{aligned}
\rho(\tau) & =\frac{E\left[x\left(t_{p}\right) x\left(t_{p}+\tau\right)\right]-E\left[x\left(t_{p}\right)\right] E\left[x\left(t_{p}+\tau\right)\right]}{\sigma_{t_{1 p}} \sigma_{t_{p}}+\tau} \\
& =\frac{N S_{12}-S_{1} s_{2}}{\sqrt{\left[N S_{11}-S_{1}^{2}\right]\left[N S_{22}-S_{2}^{2}\right]}}
\end{aligned}
$$

Where:

$$
\begin{gathered}
S_{1}=\sum_{p=0}^{N-1} x\left(t_{p}\right) \quad S_{2}=\sum_{p=0}^{N-1} x\left(t_{p}+\tau\right) \\
S_{11}=\sum_{p=0}^{N-1} x^{2}\left(t_{p}\right) \quad S_{22}=\sum_{p=0}^{N-1} x^{2}\left(t_{p}+\tau\right) \\
S_{12}=\sum_{p=0}^{N-1}\left[x\left(t_{p}\right) x\left(t_{p}+\tau\right)\right]
\end{gathered}
$$

First, the hour values of ionosphere characteristic parameters $\mathrm{z}$ are arranged to a function of stationarity time series, then linear filter is adapted, and the $\mathrm{z}$ (predicted value) at one point is expressed as weighted average of $n$ measured values.

$$
z(t)=\sum_{j=0}^{n-1} \lambda_{j} Z\left(t_{j}\right)
$$

Under the optimal and unbiased conditions, $\mathrm{Z}(\mathrm{t})$ is considered as a stationary stochastic process, where weighted coefficient $\lambda_{\mathbf{j}}$ can be obtained by the autocorrelation coefficient $\rho(t)$ of $Z(t)$. Then $\lambda_{j}$ and $\rho(t)$ meet the following linear formulas:

$$
\begin{gathered}
\sum_{i=0}^{n-1} \lambda_{j} \rho\left(t_{i}-t_{j}\right)+\mu=\rho\left(t-t_{i}\right), i=0,1,2_{v} \ldots{ }_{v} n-1 \\
\sum_{i=0}^{n-1} \lambda_{j}=1
\end{gathered}
$$

Where $\boldsymbol{\mu}$ is the Lagrange multiplication factor, and $\boldsymbol{\rho}(\tau)$ is the autocorrelation coefficient of the function $\mathrm{Z}(\mathrm{t})$.

$$
p(\tau)=\frac{M Z(t) Z(t+\tau)-(M Z)^{2}}{M Z^{2}-(M Z)^{2}}
$$

If the autocorrelation coefficient $\rho$ of $Z(t)$ is known, then these $n+1$ linear formulas can be worked out. Those answers $\lambda_{j}$ and $\mu$ will be plugged into equation (3-8), then the observed value in $\mathrm{t}$ time can be obtained.

\section{PREDICTED RESUlt ANALYSIS}

Ionospheric data in Sanya, Hainan in 2014 is adopted to predict, and different seasons are selected in one year, that is June (the Sun activity is stronger) and December (the sun activity is weaker). Seven days' ionospheric data which are known are selected to predict the eighth day's, then the predicted value is compared to the known value, and analyzed.

Ionospheric data in June and December are adopted to predict and analyze respectively, then seven days' data are randomly selected as the forecast object, and these seven days' data, autocorrelation predicted value and practical value are drawed out, compared and analyzed.

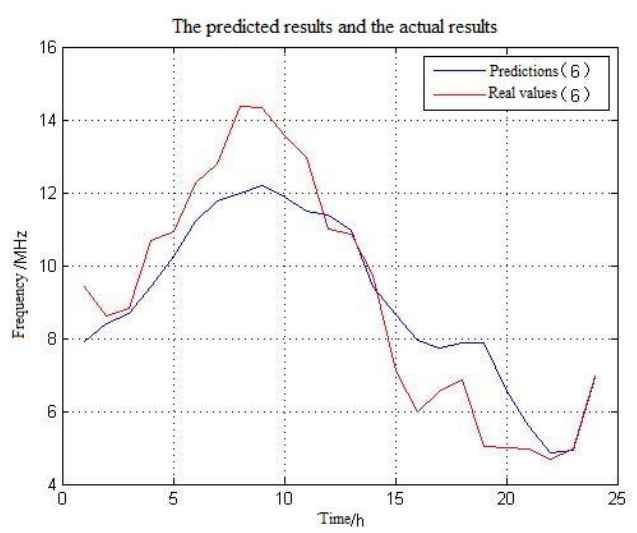

FIGURE I. PREDICTED VALUE AND ACTUAL VALUE IN JUNE

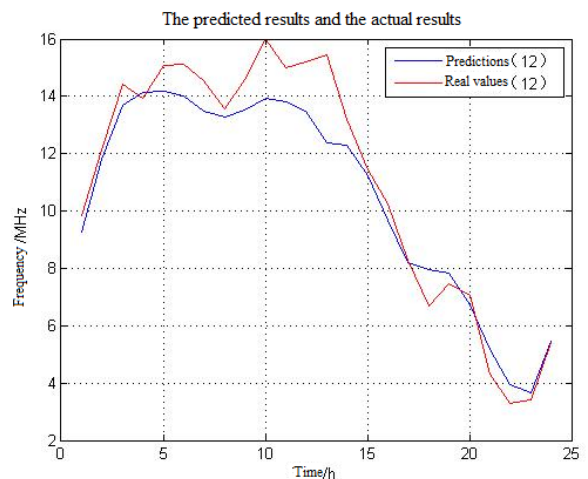

FIGURE II. PREDICTED VALUE AND ACTUAL VALUE IN DECEMBER

Comparison of these two pictures in June and December reveals that predicted value and practical value of different seasons are nearly consistent, and achieve ideal results.

To test the advantage of autocorrelation coefficient prediction method further, autocorrelation coefficient predicted foF2, artificial neural network predicted foF2 and practical foF2 are compared and analyzed, then the MUF of these foF2 in June and December are worked out to compared too. 


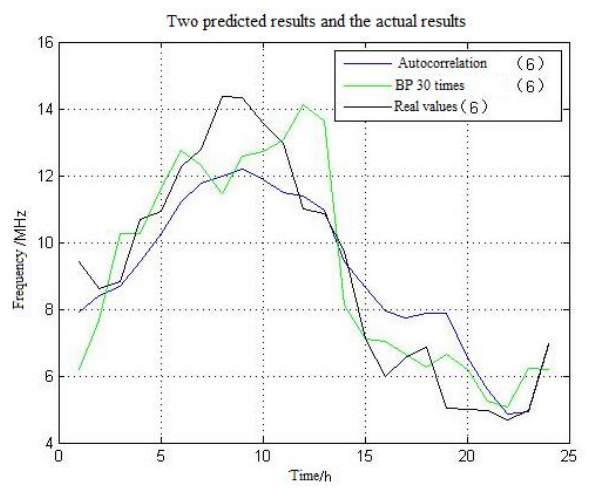

FIGURE III. PREDICTED RESULTS ANALYSIS IN JUNE

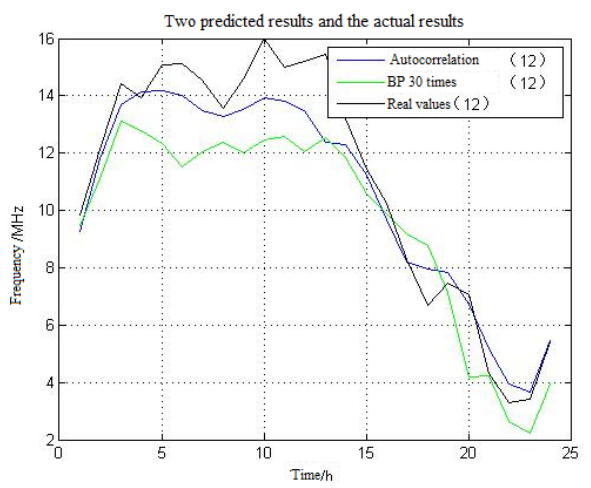

FIGURE IV. PREDICTED RESULTS ANALYSIS IN DECEMBER

FIGURE III is the compared results: the foF2 on the 12 noon of autocorrelation coefficient prediction method is $10.9643 \mathrm{MHz}$, and the corresponding MUF is $11.5143 \mathrm{MHz}$; the foF2 on the 12 noon of artificial neural network prediction method(30 times) is $13.6441 \mathrm{MHz}$, and the corresponding MUF is $14.1914 \mathrm{MHz}$; the practical foF 2 on the 12 noon is $10.8500 \mathrm{MHz}$, and the corresponding MUF is $12.4500 \mathrm{MHz}$.

Results can also be worked out from Fig.4, the MUF on the 12 noon of autocorrelation coefficient prediction method is $15.3314 \mathrm{MHz}$, and the MUF on the 12 noon of artificial neural network prediction method is $14.3149 \mathrm{MHz}$, and the practical MUF on the 12 noon is $16.2761 \mathrm{MHz}$.

Finally, we arrive at the conclusion by calculation and analysis, the predicted data with autocorrelation coefficient method is close to the practical frequency than artificial neural network method.

\section{REFERENCES}

[1] MUHTAROV P, KUTIEV I. Autocorrelation method for temporal interpolation and short-term prediction of ionospheric data[J]. Radio Science, 1999, 34(2): 459-464.

[2] MUHTAROV P, KUTIEV I, CANDER L. Geomagnetically correlated autoregression model for shortterm prediction of ionospheric parameters[J]. Inverse Problems, 2002. 18(1): 49-65.

[3] MIKHAILOV A V, MORENA B A DE LA, MIRO G. A method for foF2 monitoring over Spain using the EI Arenosillo digiSonde current obsenvations[M]. 3rd COST 251 Workshop proceedings. EI Arenosillo. Spain, 1998, COST251TD(99)003, 185-194.

[4] MARIN D, MIRO G, MIKHAILOV A V. A method for foF2 short-term prediction, 4th COST 251 Workshop preceedings[M]. Madeira, Portugal, 1999. COST251TD(99)008, 214-222.
[5] LUNDSTEDT H. Neural network and prediction of solar terrestrial effects[J]. Planet Space Sci., 1992, 40(4): 457-464

[6] CANDER L J R, MiLOSAVLJEVIC M. STANKOVIC S. et al. Ionospheric forecasting technique by artificial neural net-work[J]. Electron Lett, 1998, 34(6): 1573-1574.

[7] KONG Qingyan, LIU Wen, JIAO Peinan, et al. Twenty_four hour ahead prediction of foF2[J]. Chinese Journal of Space Science, 2009, 29(4): 377-382. (in Chinese)

[8] FENG Jing, LIU Wen, FAN Junmei, et al. A New Method for Shortterm Ionospheric Forecast[J]. Equipment Environmental Engineering, 2009, 6(3): 15-20. (in Chinese)

[9] LIU Wen, JIAO Peinan, FENG Jing, et al. Method of similar days for ionospheric parameter short-term forecasting[J]. Chinese Journal of Radio Science, 2010, 25(2): 240-247. (in Chinese) 